\title{
IL6 Receptor Facilitates Adipogenesis Differentiation of Human Mesenchymal Stem Cells through Activating P38 Pathway
}

\author{
Wen Deng ${ }^{1, *}$, Huadi Chen ${ }^{2, *}$, Hongjun $\mathrm{Su}^{1, *}$, Xiaohua $\mathrm{Wu}^{1}$, \\ Zhongyu Xie ${ }^{1,3}$, Yanfeng $\mathrm{Wu}^{1}$, Huiyong Shen ${ }^{3}$ \\ ${ }^{I}$ Center for Biotherapy, Sun Yat-sen Memorial Hospital, Sun Yat-sen University, Guangzhou, China \\ ${ }^{2}$ Organ Transplant Center, First Affiliated Hospital, Sun Yat-sen University, Guangzhou, China \\ ${ }^{3}$ Department of Orthopedics, Sun Yat-sen Memorial Hospital, Sun Yat-sen University, Guangzhou, China
}

Background and Objectives: Mesenchymal stem cells (MSCs) have the multipotent capacity to differentiate into multiple tissue lineages as well as to self-renew, which is the main origin of adipocytes. IL6/IL6R pathway exerts a significant role in tissue regeneration and cell differentiation. Whereas, the underlying mechanism between IL6/IL6R pathway and MSCs adipogenesis differentiation remains elusive.

Methods: MSCs from healthy donors were cultured in adipogenesis differentiation medium for $0 \sim 14$ days, during which their adipogenesis differentiation degree was evaluated by Oil Red O staining. The expression of IL6R was detected in MSCs during adipogenesis differentiation. Knockdown and overexpression of IL6R were respectively performed using siRNA and lentivirus to investigate its effect on MSCs adipogenesis differentiation. The adipogenesis marker genes expression and MAPK pathway activation were detected by Western blotting. The role of P38 pathway in the adipogenesis differentiation of MSCs was determined using the specific inhibitor SB203580.

Results: The expression of IL6 and IL6R increased during adipogenesis differentiation in MSCs, which were positively correlated with Oil Red O quantification result. Knockdown and overexpression experiments demonstrated a positive correlation between the expressions of IL6R and MSCs adipogenesis differentiation, accompanied by same trend of P38 phosphorylation. Besides, the specific P38 inhibitor SB203580 markedly inhibited the adipogenesis differentiation potential of MSCs.

Conclusions: This study reveals IL6R facilitates the adiogenesis differentiation of MSCs via activating P38 pathway.

Keywords: Mesenchymal stem cells (MSCs), Adipogenesis differentiation, Interleukin 6 (IL6), Interleukin 6 receptor (IL6R), P38

Received: May 29, 2019, Revised: August 30, 2019, Accepted: September 16, 2019, Published online: December 31, 2019

Correspondence to Yanfeng Wu

Center for Biotherapy, Sun Yat-sen Memorial Hospital, Sun Yat-sen University, 107 Yan Jiang West Road, Guangzhou 510315, China

Tel: +86-020-81-332-612, Fax: +86-020-81-332-612, E-mail: swzlzx8888@126.com

Co-Correspondence to Huiyong Shen

Department of Orthopedics, Sun Yat-sen Memorial Hospital, Sun Yat-sen University, 107 Yan Jiang West Road, Guangzhou 510315, China

Tel: +86-020-81-332-199, Fax: +86-020-81-332-199, E-mail: shenhuiy@yeah.net

*These authors contributed equally to this work.

(a) This is an open-access article distributed under the terms of the Creative Commons Attribution Non-Commercial License (http://creativecommons.org/licenses/by-nc/4.0/), which permits unrestricted non-commercial use, distribution, and reproduction in any medium, provided the original work is properly cited.

Copyright (c) 2020 by the Korean Society for Stem Cell Research 


\section{Introduction}

Mesenchymal stem cells (MSCs) are special cells, which can be isolated from the bone marrow aspirates and many other tissues, and can be expanded in vitro as well as differentiate into various kinds of mesoderm-lineages (1). When cultured in specific inducing medium, MSCs reveal its osteogenic, chondrogenic or adipogenic potential. Since MSCs were proved to be a major source of adipocyte, the adipogenesis of MSCs affects the development of obesity and metabolic syndrome in various ways, and therapies based on MSC aiming to treat obesity and its commodities are raising increasing concern (2). For instance, Lee CW et al. developed several MSC-based therapies which reduced obesity and metabolic disorders on rodents (3).

Although IL6 is a well-known pleiotropic cytokine, its receptor is expressed only in limited types of cells, including hepatocytes, leukocytes megakaryocytes and so on, and its function varies when expressed in different cells or activated in different time phase or pattern (4). Its effects on regulating inflammation and activity of immune cells have received much attention in previous studies, while its hormone-like influence on many other cells is revealed recently as well. IL6/IL6R and its downstream signaling pathways play an important role in the development of obesity and metabolic syndrome. High level secretion of cytokines such as IL6 is a sign of chronic inflammation, which is an essential mechanism of obesity and metabolic syndrome (5). IL6 level is found elevated among the patients with lipid metabolic disorders and insulin resistance (6). Pradhan et al. (7) proved that high serum IL6 level was significantly correlated to higher relative risk of diabetes mellitus in female. Theurich et al. (8) described that IL6R highly expressing NK cell is correlated with systemic low-grade inflammation and higher level of insulin resistance. As for MSCs, IL6R was found expressed on MSCs and contributed to various biological behaviors of MSCs, such as self-renewal (9), osteogenic differentiation (10) and chondrogenic differentiation (11). Our previous study has reported that IL6/IL6R pathway promotes osteogenic differentiation of mesenchymal stem cells, which is mediated by stat3-dependently downstream (10). And the connections between the regulation of MSC adipogenic differentiation and other pathways were more or less revealed, such as Wnt, Hedgehod, NELL-1, BMP and IGF (12), but the connection between IL6/IL6R pathway and MSC adipogenic differentiation, and the related downstream signals remain unknown.

This study is to explore the correlation between IL6/
IL6R pathway and MSC adipogenic differentiation. In this study, we investigated whether IL6R facilitates adipogenic differentiation of MSCs and made a preliminary exploration on the downstream pathway that involves the regulation. We firstly induced adipogenic differentiation of MSCs and detected both the generation of triglycerol by Oil Red dyeing and the expression of PPAR- $\gamma$ and CEPB- $\alpha$, the significant trancription factors of adipogenesis, by Western blotting, to determine the effect of the induction. During the induction, IL6R was knocked down or overexpressed, and at the same time the effect of adipogenic differentiation was observed and compared to the negative control group. Also, the activation of P38, JNK and ERK pathways were monitored by Western blotting, in order to locate the downstream pathway of IL6R regulating the adipogenesis of MSCs. This study demonstrates that IL6R contributes to the adiogenic differentiation of MSCs by activating P38 pathway.

\section{Materials and Methods}

\section{MSCs isolation and culture}

MSCs were isolated and purified as previously reported from the fresh bone marrow aspiration samples from healthy donors (13), and seeded in 12-well plates and cultured in Dulbecco's modified Eagle's medium (DMEM; Gibco) with 10\% fetal bovine serum (FBS; Sijiqing Biological Engineering Material Company) at $37^{\circ} \mathrm{C} / 5 \% \mathrm{CO}_{2}$. Medium was replaced every 3 days. MSCs were passaged at about $90 \%$ confluence and the fourth passages were used for the experiments. Informed consent was signed by each healthy donors before participation. This study was reviewed and approved by the ethics committee of Sun Yat-Sen Memorial Hospital, Sun Yat-Sen University, Guangzhou, China.

\section{Flow cytometric analysis}

MSCs were harvested and incubated with anti-CD29, CD44, CD105 or CD14, CD45, HLA-DR antibody (BD Biosciences, New York, USA) for 30 minutes at $4^{\circ} \mathrm{C}$. Then the cells were washed through centrifugation and analyzed by flow cytometry Coulter Flow Cytometer system (BD Biosciences, New York, USA).

\section{Triple differentiation potentials assay}

Adipogenic differentiation: MSCs were maintained in adipogenesis differentiation medium (DMEM, 10\% FBS, $10 \mathrm{mg} / \mathrm{ml}$ insulin (Sigma-Aldrich), $0.5 \mathrm{mM}$ 3-isobutyl-1methylxanthine (Sigma-Aldrich), $1 \mathrm{mM}$ dexamethasone 
(Sigma-Aldrich), $100 \mathrm{IU} / \mathrm{ml}$ streptomycin (Sigma-Aldrich) and $100 \mathrm{IU} / \mathrm{ml}$ penicillin (Sigma-Aldrich)) for $0 \sim 14$ days. Adipogenesis differentiation was evaluated by Oil Red O staining.

Osteogenesis differentiation: MSCs were cultured in osteogenesis differentiation medium (DMEM, 10\% FBS, $50 \mu \mathrm{M}$ ascorbic acid (Sigma-Aldrich), $0.1 \mu \mathrm{M}$ dexamethasone, $10 \mathrm{mM} \beta$-glycerol phosphate, $100 \mathrm{IU} / \mathrm{ml}$ streptomycin (Sigma-Aldrich) and $100 \mathrm{IU} / \mathrm{ml}$ penicillin (SigmaAldrich)). Osteogenesis differentiation was assessed by Alizarin Red staining on day 14.

Chondrogenic differentiation: MSCs were grown in chondrogenic differentiation medium (high-glucose DMEM, 1\% ITS Premix (Corning Life Sciences, Wisconsin, USA), $1 \mathrm{mM}$ sodium pyruvate (Sigma-Aldrich), $100 \mathrm{nM}$ dexamethasone, $50 \mathrm{mg} / \mathrm{L}$ ascorbic acid and $10 \mathrm{ng} / \mathrm{ml} \mathrm{recombi-}$ nant human TGF- $\beta 3$ (R\&D Systems, Minnesota, USA). Adipogenesis differentiation was detected by Alcian Blue staining on day 21 .

\section{Oil Red 0 staining and quantification}

After washed by phosphate-buffered saline (PBS), MSCs were fixed in 10\% formalin and stained with Oil Red O working solution for $30 \mathrm{~min}$ and then were washed by PBS for three times again. At last, the stained cells were observed under microscope. Lipid droplets were extracted with isopropanol from the stained cells and the optical density was measured with a spectrophotometer at 490 nm.

\section{Western blotting}

MSCs were lysed, extracted and then boiled with loading buffer. Equal amounts of protein were loaded into 10\% SDS-PAGE for seperation and transferred to PVDF membranes (Millipore). The membranes were blocked in skimmed milk powder and incubated with primary antibodies against GAPDH, IL6R, CEBP- $\alpha$, PPAR- $\gamma$, P38 MAPK, phosphorylated P38 MAPK, ERK, phosphorylated ERK, JNK and phosphorylated JNK (each diluted 1 : 1,000; Cell Signaling Technology) at $4^{\circ} \mathrm{C}$ overnight. After washing for 3 times, the membranes were treated with a horseradish peroxidase (HRP)-conjugated secondary antibody (diluted 1 : 3,000; Santa Cruz Biotechnology). Specific antibody-antigen complexes were detected and visualized by Immobilon Western Chemiluminescent HRP Substrate (Millipore). The intensities of all bands were quantified by ImageJ software (National Institutes of Health, Bethesda, MD, USA).

\section{Enzyme-linked immunosorbent assay (ELISA)}

Cell culture supernatants were collected when changing medium at the indicated time points. Expression of IL6 in culture supernatants was detected using Human IL-6 QuantikineELISA Kits (R\&D Systems) according to the manufacturer's instructions.

\section{Lentivirus construction and infection}

The IL6R siRNA was designed and synthesized by GenePharma (Shanghai, China). One siRNA with the best knockdown efficiency was selected to build the short hairpin RNA (shRNA) and construct the lentivirus with a target sequence of $5^{\prime}-G G A A G A C A A T G C C A C T G T T C A-3^{\prime}$. The IL6R overexpression lentiviruses were generated by co-transfecting pGLVH1/GFP/Puro (Gene Pharma) and packing plasmids (pGag/Pol, pRev, and pVSV-G) into 293T cells. Culture supernatants containing lentiviruses were strained and concentrated $72 \mathrm{~h}$ after transfection. Each lentivirus (109 transducing units/ml) and polybrene $(5 \mathrm{mg} / \mathrm{ml}$ ) were added into the medium and incubated with MSCs for $24 \mathrm{~h}$ at a multiplicity of infection of 50 . Then the medium was replaced with the adipogenic differentiation medium. The differentiated MSCs were used for the related experiments.

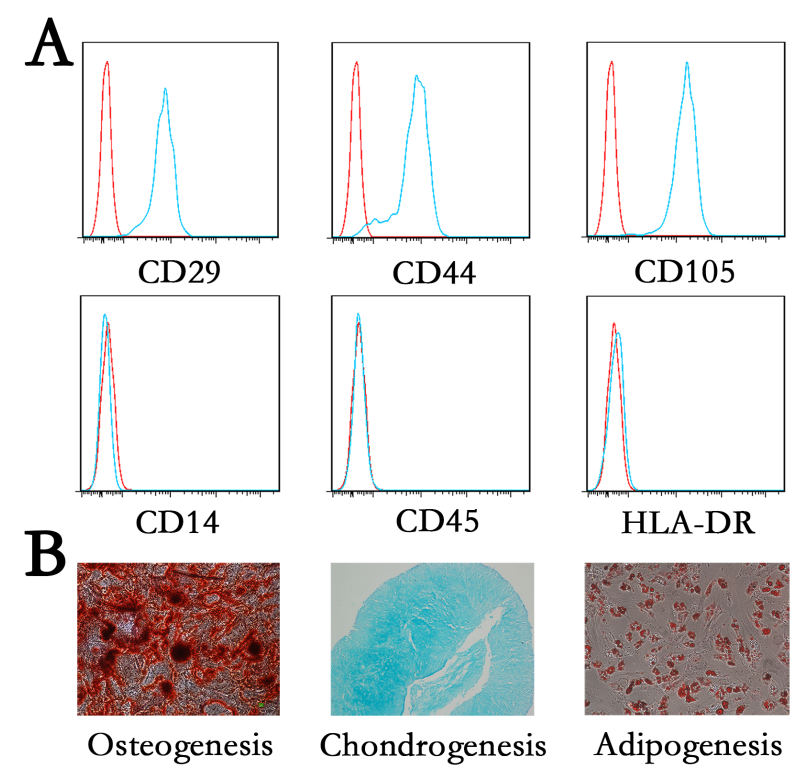

Fig. 1. Immunophenotype investigation and induced differentiation of MSCs. (A) MSCs were positive for CD29, CD44, CD90 and CD105 and negative for CD14, CD45, and HLA-DR. (B) MSCs was induced to differentiate into osteoblasts, chondrocytes and adipocytes. 


\section{Exogenous inhibition assay}

SB203580, a specific p38 signaling pathway inhibitor, was added at a concentration of $25 \mu \mathrm{M}$ during the adipogenesis differentiation of MSCs.

\section{Statistical analysis}

All data were expressed as the mean \pm SD. Unpaired
Student's $\mathrm{t}$ test and Pearson correlation test were performed for statistical analyses using SPSS Version 22.0 (IBM SPSS Inc., Armonk, NY, USA). p-values $<0.05$ were considered significant.

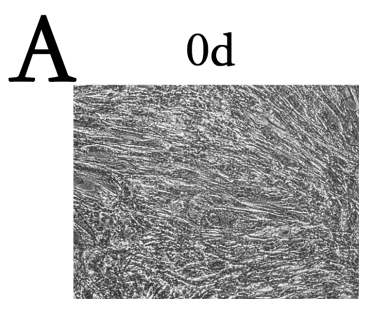

$10 \mathrm{~d}$

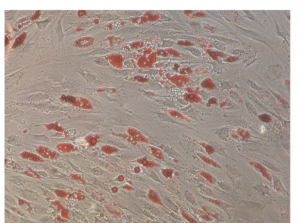

Quantification of Oil Red O

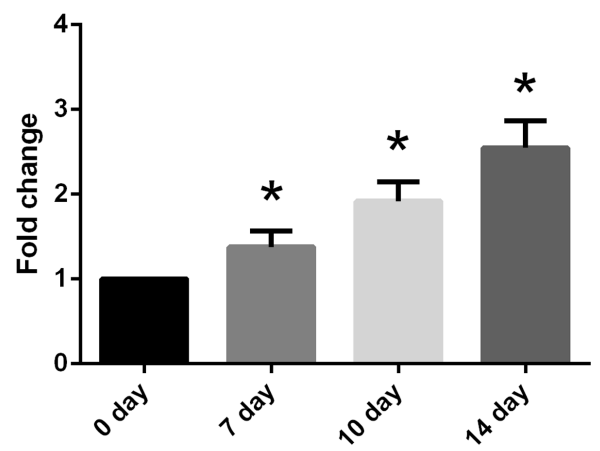

Days of induction

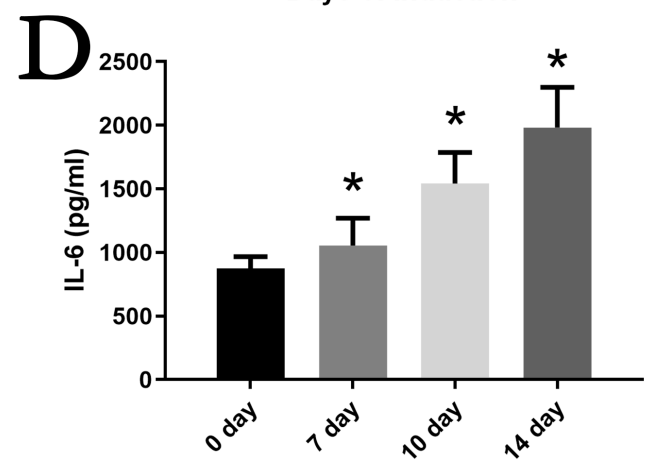

Days of induction
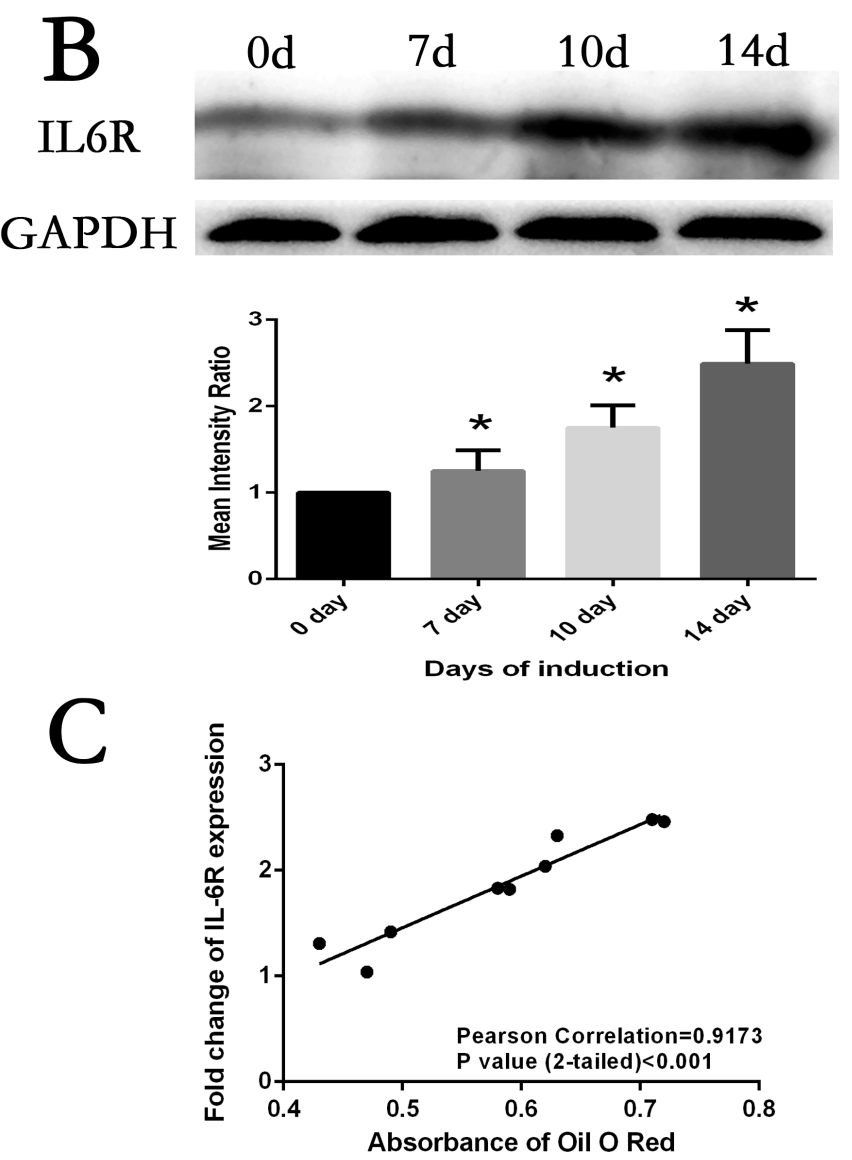

$\mathrm{E}$

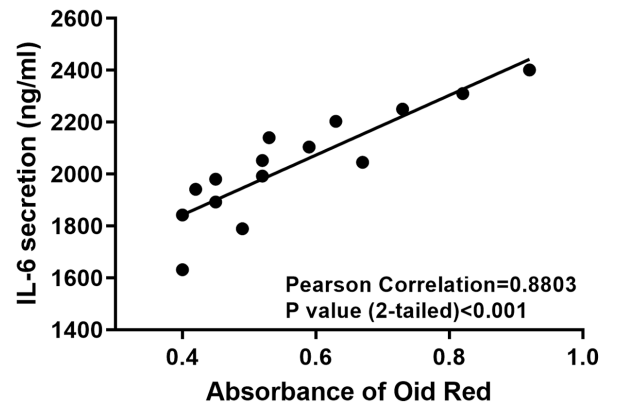

Fig. 2. Correlation between MSCs adipogenesis differentiation and IL6R. (A) MSCs adipogenic differentiation from day0 to day14 was assessed by Oil red $\mathrm{O}$ staining and quantification. (B) IL6R expression was significantly increased from day0 to day14 during induction. (C) IL6R expression was positively correlated with Oil red O staining results in MSCs. (D) IL6 secretion was significantly increased from day0 to day 14 during induction. (E) IL6 secretion was positively correlated with Oil red O staining results in MSCs. Data is displayed as the means $\pm \mathrm{SD} .{ }^{*}$ represents $\mathrm{p}<0.05$. 


\section{Results}

\section{Immunophenotype investigation and induced differentiation of MSCs}

To determine the purity and phenotypic features of MSCs used in our study, we invsetigated specific cell surface markers of MSCs by flow cytometer analysis. Cultured in self-renewal medium without induced differentiation, MSCs highly expressed positive markers CD29, CD44 and CD105 and were negative for CD14, CD34, and CD45 (Fig. 1A). After treated with adipogenesis, osteogenesis and chondrogenesis medium at day21, day14 and day14 respectively, differentiation degree of MSCs were assessed by Alizarin Red, Alcian blue and Oil Red O staining (Fig. 1B). These data indicated that MSCs display unique immunophenotype $(\mathrm{CD} 29+\mathrm{CD} 44+\mathrm{CD} 105+\mathrm{CD} 14-$ CD34-CD45+) and are multipotent cells that can differentiate into osteoblasts, chondrocytes and adipocytes.

\section{Correlation between MSCs adipogenesis differentiation and IL6/IL6R}

MSCs were maintained in adipogenesis medium to induce adipogenesis differentiation. At day7, day10 and day14, differentiation status of MSCs was evaluated by Oil Red O staining. As shown in Fig. 2A, lipid accumulation was visualizing increasedly with extension of culture time, which was confirmed by comparing the quantification of
Oil Red O staining. Simultaneously, the protein expression of IL6R of adipogenesis MSCs was detected. The result of Western Blot analysis demonstrated IL6R level was steadily upregulated (Fig. 2B). Of note, we found a significant correlation between the levels of lipid accumulation and IL6R during adipogenesis differentiation of MSCs $(r=0.9173, p<0.001)$ (Fig. 2C). The levels of IL6 in cell culture supernatants exhibited the same trend as IL6R protein expression (Fig. 2D), which also positively correlated with Oil Red O quantification result $(\mathrm{r}=0.8803$, $\mathrm{p}<0.001$ ) (Fig. 2E).

\section{SiRNA knockdown of IL6R inhibited adipogenesis differentiation of MSCs}

To analyze the role of the IL6R during adipogenesis differentiation, gene knockdown of IL6R was performed in MSCs. MSCs were cultured in adipogenesis medium to induce adipogenesis differentiation for 10 days. The ability of adipogenesis differentiation was assessed by visualizing lipid accumulation. The microscopic observation and quantification results of Oil Red O staining revealed that downregulation of IL6R by its specific siRNA could significantly suppress the process of adipogenesis differentiation. The total lipid accumulation was largely reduced to $50 \%$ in the knockdown group when compared with the normal control and induction group (Fig. 3A, 3B). At the same time, these cells were harvested to detect the protein
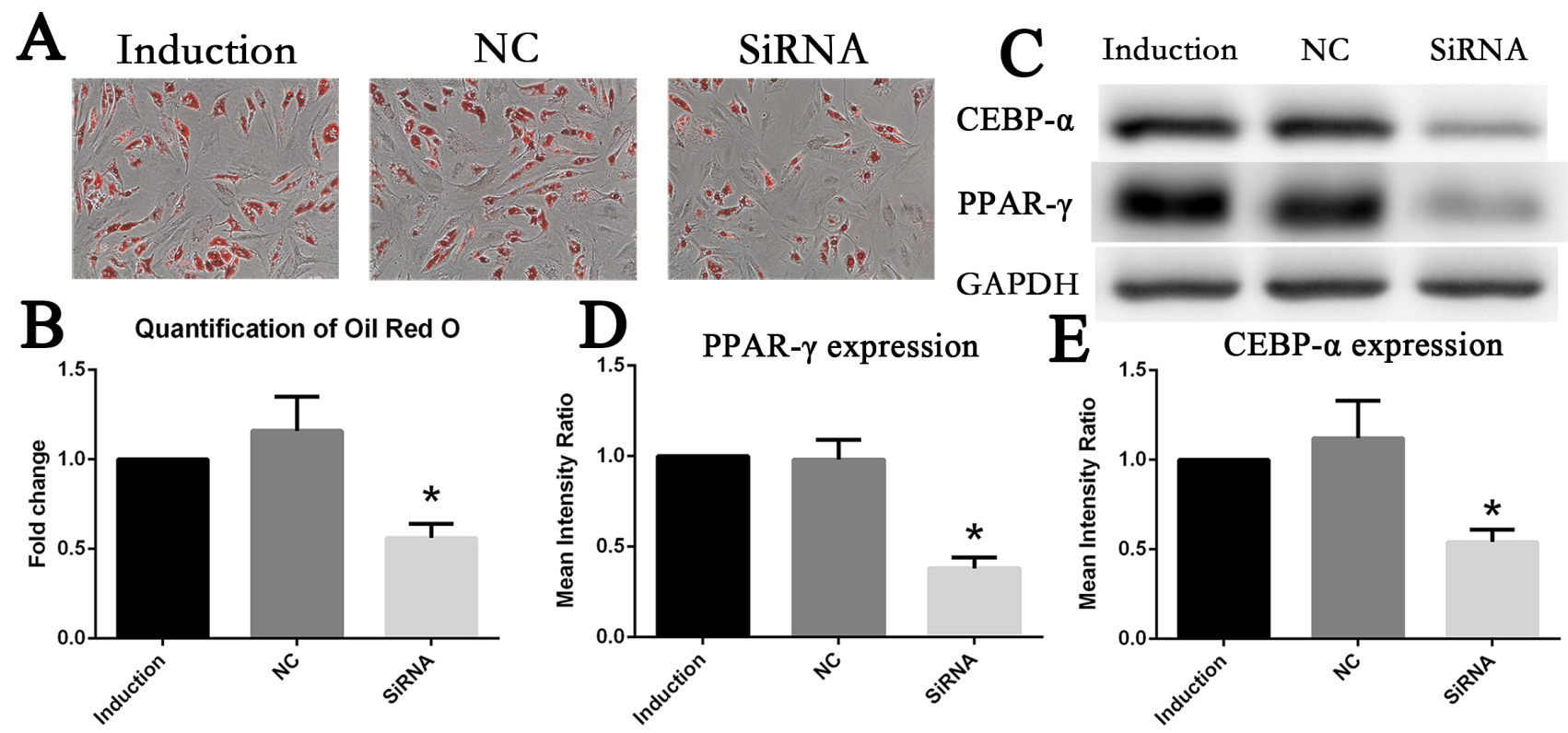

Fig. 3. Downregulation of IL6R inhibited adipogenesis differentiation of MSCs (A, B) The ability of adipogenesis differentiation of MSCs decreased after IL6R siRNA knockdown. (C $\sim$ E) Decreased expression of MSCs adipogenic differentiation marker genes CEBR- $\alpha$ and PPAR- $\gamma$ after knockout of IL6R. * represents $\mathrm{p}<0.05$. 
expression levels of pivotal adipogenesis transcription factors. Consistent with lipid accumulation levels, in the siRNA group, the protein levels of PPAR- $\gamma$ and CEBP- $\alpha$ were descending to roughly half of that in the control groups (Fig. 3C $\sim \mathrm{E}$ ).

\section{Overexpression of IL6R enhanced adipogenesis differentiatability of MSCs}

Conversely, gene overexpression of IL6R was performed in MSCs by transfecting with lentiviral plasmid. At day10 of adipogenesis induction, Oil Red $\mathrm{O}$ staining revealed that lipid accumulation level of MSCs in the upregulation group significantly ascended compared with adipogenesis MSCs without treatment (Fig. 4A, 4B), which indicated upregulation of IL6R could foster adipogenesis differentiation of MSCs. It was furthermore confirmed that expression of PPAR- $\gamma$ and CEBP- $\alpha$ were enhanced to more than two fold of control groups in overexpression group (Fig. $4 \mathrm{C} \sim \mathrm{E}$ ).

\section{Involvement of P38 phosphorylation in the adipogenesis differentiation-enhancing effect of IL6R}

To figure out whether IL6R could activate MAPK pathways during adipogenesis differentiation of MSCs, we examined the expression and phosphorylation levels of P38, ERK and JNK in the control groups, siRNA group and overexpression group of IL6A respectively. As shown by Fig. 5A and 5C, the expression levels and phosphorylation levels of ERK and JNK showed no significant difference in all groups, while P38 was significantly decresed when IL6R was knocked down compared with the control groups. On the contrary, in the overexpression group, only P38, especially p-P38, was obviously increased (Fig. 5B, 5D).

These results imply that the phosphorylation of P38 plays an important role in the adipogenesis differentiation-boosting effects of IL6R.

\section{Discussion}

In the present study, we firstly found that increased IL6 secretion and IL6R expression during adipogenesis differentiation of MSCs, which had positve correlation with lipid accumulation. By performing knockdown and overexpression of IL6R, we observed that adipogenesis differentiation was correspondingly repressed and promoted accompanied by decrease and increase of lipid accumulation and significant trancription factors, PPAR- $\gamma$ and CEPB- $\alpha$ (14). Moreover, we noticed P38 MAPK pathway showed the same trend of activation and inactivation when knockdown and overexpression of IL6R, and that the specific P38 inhibitor SB203580 apparently suppressed the adipo-

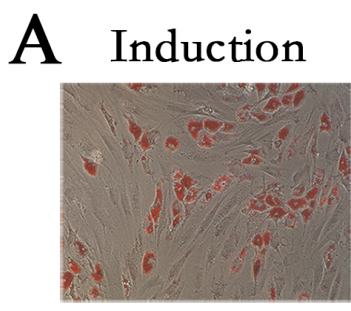

B

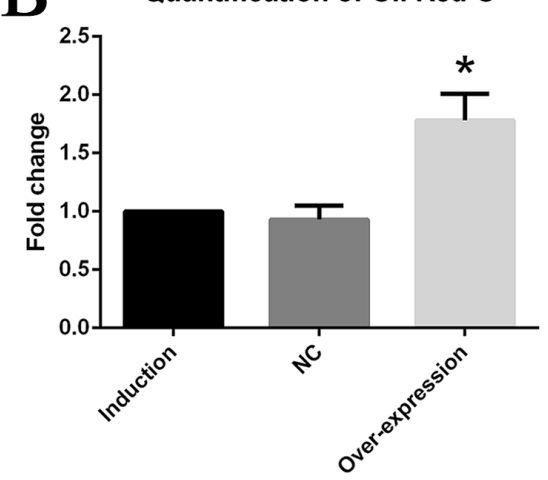

NC

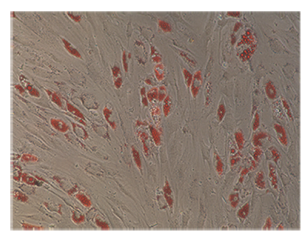

D

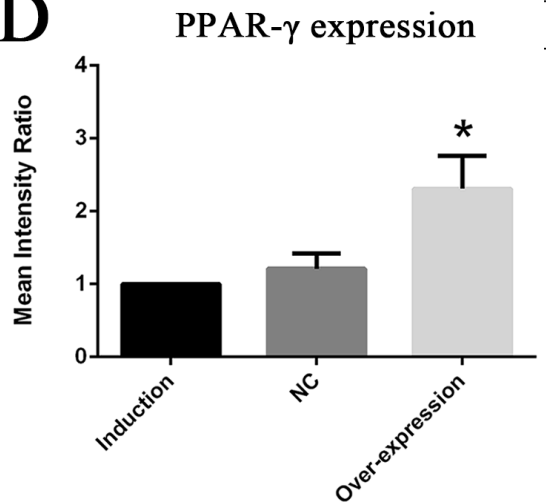

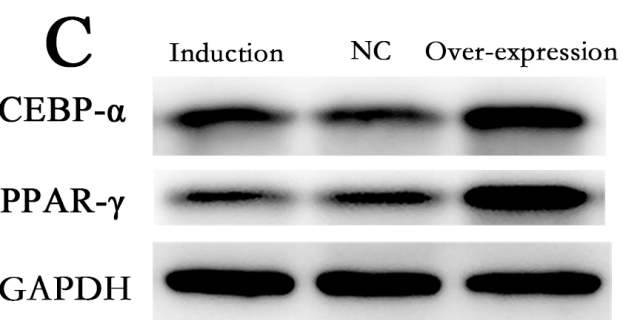

$\mathrm{E}$

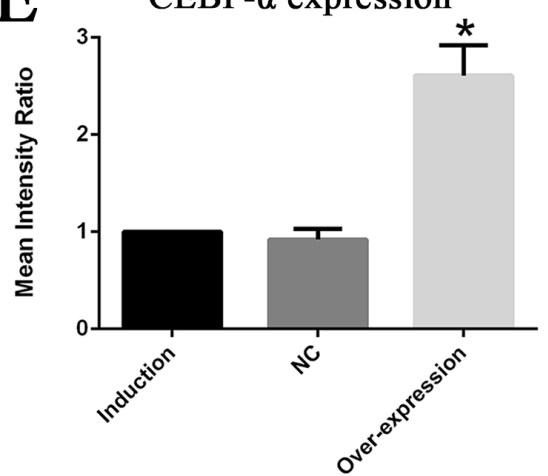

Fig. 4. Upregulation of IL6R enhanced adipogenesis differentiation of MSCs (A, B) The adipogenesis differentiation of MSCs was enhanced after IL6R overexpression. ( $\sim$ E) Enhanced expression of MSCs adipogenic differentiation marker genes CEBR- $\alpha$ and PPAR- $\gamma$ after IL6R overexpression. ${ }^{*}$ represents $\mathrm{p}<0.05$. 


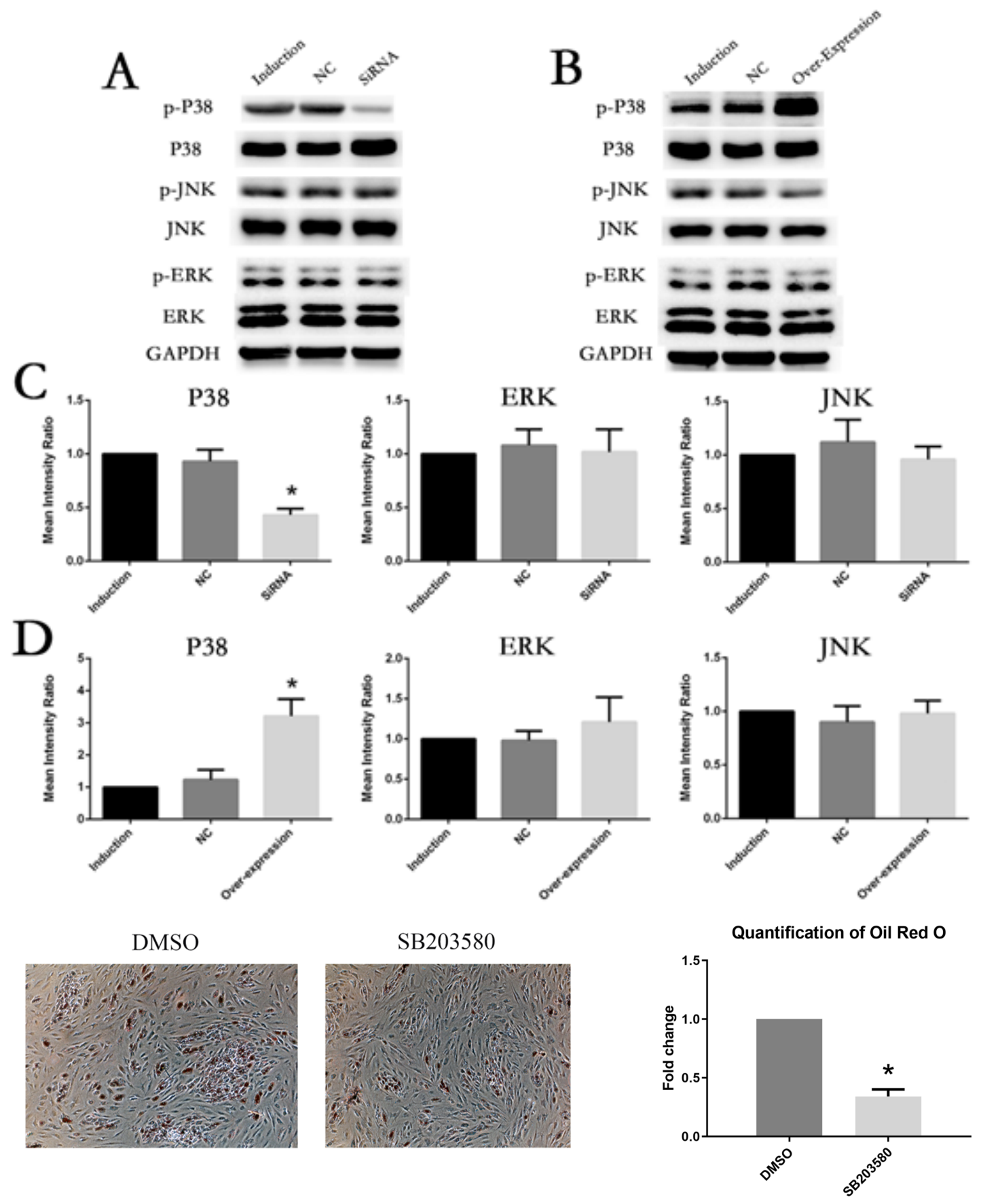

Fig. 5. Involvement of P38 phosphorylation in the adipogenesis differentiation-enhancing effect of IL6R. (A, C) After IL6R knockout, the activation of P38 pathway was inhibited and there was no significant change in ERK or JNK. (B, D) After IL6R overexpression, the activation of P38 pathway was enhanced, and ERK as well as JNK showed no significant changes. (E) Oil Red O staining on day 10 of adipogenesis differentiation was significantly reduced by SB203580. * represents $\mathrm{p}<0.05$.

genesis differentiation potential of MSCs. Therefore, our results indicate that IL6R facilitates adipogenic differentiation by activating P38 pathway.

IL6R is a type I cytokine receptor that binds IL6 to exert pleiotropic effect (15). Previous studies mainly foucus their role on the immune cells, but now they are appreciated to have hormone-like influence on many other cells as well (4). MSCs have great differentiation capacity and can differentiate into diverse cell types, including osteoblasts, chondrocytes and adipocytes (16). In our study, 
these cells are characterized by distinct immunophenotype (CD29+CD44+CD105+CD14-CD34-CD45-), which is consistent with previous report $(16,17)$. Xie et al. (10) reported that IL6/IL6R expression level ascends during differentiation and can accelerate osteogenic differentiation in MSCs. Of note, MSCs can spontaneously generate abundant amounts of IL6 in the culture supernatant, which maintains their stemness and skews to self-renewal without differentiation (9). In addition, Kondo et al. (11) demonstrated that IL6R $\alpha$ expression increases in the progression of differentiation and positvely regulates the chondrogenesis differentiation. In our study, we revealed that the IL6 and IL6R produced by MSCs were upregulated during adipogenesis differentiation, which further enhanced adipogenesis differentiation. These results were mainly in compliance with above-mentioned reports. We conclude that different culture conditions might give rise to the different effect of IL6/IL6R.

MAPK signaling constitutes three major subfamilies, including ERK, JNK and P38, which are involved in various physiological and pathophysiological processes, including cell and tissue growth, differentiation, apoptosis and inflammation (18). Legendre et al. (19) proved that IL6/ IL6R activated ERK1/2 MAPK in bovine chondrocytes. Besides, Chen et al. (20) demonstrated that ICAM-1 facilitated adipogenesis of murine MSC cell line by activating P38 pathway. Of note, Pricola et al. (21) reported that IL6 promoted MSCs proliferation and inhibited differentiation depending on ERK1/2 activation. Based on the above reports, we found regulation of IL6R to adipogenesis differentiation was dependent on phosphorylation of P38, instead of ERK and JNK in the present study, which is consistent with the report by Aouadi et al. (22) that p38MAP Kinase activity is required for human primary adipocyte differentiation. There ara many reports that inhibition of $\mathrm{p} 38 \mathrm{MAPK}$ increases adipogenesis in mouse embryonic fibroblast and preadipocytes $(23,24)$. But the mouse embryonic fibroblast and preadipocytes are of great differnece from the cells, bone MSCs donating by healthy volunteers, in our study. Besides, the induction culture condition is different. We speculate that different cell types may have different effects in response to different culture conditions. The trilineage differentiation of MSCs is regulated by unique pathways and, obviously, P38 MAPK pathway plays an significant part in adipogenesis differentiation. It is reported that disruption of actin polymerization inhibited osteogeneic differentiation of MSCs through P38 MAPK pathway and actin alteration is a precondition to MSCs differentiation (25), which may be the aim of future studies how IL6/IL6R regulate P38
MAPK during adipogenesis differentiation.

Nevertheless, this study has several limitations. The mechanisms how IL6/IL6R are regulated during adipogenic differentiation of MSCs are still unknown. The mechanisms how different culture conditions might give rise to the different effect of IL6/IL6R to MSCs. These issues require further study.

Fatty tissue constitutes a large amount of biologically active tissue in human body $(26,27)$. Adipocytes, mainly derived from MSCs, are the essential components of fatty tissue. Metabolic syndrome is considered to be related to the chronic inflammation involved IL6/IL6R pathway (5) and adipogenesis differentiation of MSCs (28). In conclusion, we have demonstrated that IL6R facilitates adipogenic differentiation of MSCs via activating P38 MAPK pathway. It provides a new sight to figure out the underlying mechanism how MSCs differentiate into adipocytes, which might help to prevent and treat the obesity and metabolic syndrome.

\section{Potential Conflict of Interest}

The authors have no conflicting financial interest.

\section{Author Contributions}

WD, HC and HS: design, execution of experiments, interpretation of data and writing the manuscript. XW and ZX: execution of experiments and interpretation of data and critical revision of the manuscript. YW and HS: supervision of the project, design, interpretation of data and writing of the manuscript.

\section{References}

1. Jackson WM, Nesti LJ, Tuan RS. Concise review: clinical translation of wound healing therapies based on mesenchymal stem cells. Stem Cells Transl Med 2012;1:44-50

2. Matsushita K, Dzau VJ. Mesenchymal stem cells in obesity: insights for translational applications. Lab Invest 2017;97: 1158-1166

3. Lee CW, Hsiao WT, Lee OK. Mesenchymal stromal cell-based therapies reduce obesity and metabolic syndromes induced by a high-fat diet. Transl Res 2017;182:6174.e8

4. Hunter CA, Jones SA. IL-6 as a keystone cytokine in health and disease. Nat Immunol 2015;16:448-457

5. Ouchi N, Parker JL, Lugus JJ, Walsh K. Adipokines in inflammation and metabolic disease. Nat Rev Immunol 2011;11:85-97

6. Pickup JC, Mattock MB, Chusney GD, Burt D. NIDDM as a disease of the innate immune system: association of acute-phase reactants and interleukin- 6 with metabolic syndrome X. Diabetologia 1997;40:1286-1292 
7. Pradhan AD, Manson JE, Rifai N, Buring JE, Ridker PM. C-reactive protein, interleukin 6, and risk of developing type 2 diabetes mellitus. JAMA 2001;286:327-334

8. Theurich S, Tsaousidou E, Hanssen R, Lempradl AM, Mauer J, Timper K, Schilbach K, Folz-Donahue K, Heilinger C, Sexl V, Pospisilik JA, Wunderlich FT, Brüning JC. IL-6/Stat3-Dependent induction of a distinct, obesity-associated NK cell subpopulation deteriorates energy and glucose homeostasis. Cell Metab 2017;26:171184.e6

9. Ng J, Hynes K, White G, Sivanathan KN, Vandyke K, Bartold PM, Gronthos S. Immunomodulatory properties of induced pluripotent stem cell-derived mesenchymal cells. J Cell Biochem 2016;117:2844-2853

10. Xie Z, Tang S, Ye G, Wang P, Li J, Liu W, Li M, Wang S, Wu X, Cen S, Zheng G, Ma M, Wu Y, Shen H. Interleukin-6/interleukin-6 receptor complex promotes osteogenic differentiation of bone marrow-derived mesenchymal stem cells. Stem Cell Res Ther 2018;9:13

11. Kondo M, Yamaoka K, Sakata K, Sonomoto K, Lin L, Nakano K, Tanaka Y. Contribution of the Interleukin-6/STAT-3 signaling pathway to chondrogenic differentiation of human mesenchymal stem cells. Arthritis Rheumatol 2015;67:1250-1260

12. James AW. Review of signaling pathways governing MSC osteogenic and adipogenic differentiation. Scientifica 2013;2013:684736

13. Xie Z, Wang P, Li Y, Deng W, Zhang X, Su H, Li D, Wu $\mathrm{Y}$, Shen H. Imbalance between bone morphogenetic protein 2 and noggin induces abnormal osteogenic differentiation of mesenchymal stem cells in ankylosing spondylitis. Arthritis Rheumatol 2016;68:430-440

14. Farmer SR. Transcriptional control of adipocyte formation. Cell Metab 2006;4:263-273

15. Yamasaki K, Taga T, Hirata Y, Yawata H, Kawanishi Y, Seed B, Taniguchi T, Hirano T, Kishimoto T. Cloning and expression of the human interleukin-6 (BSF-2/IFN beta 2) receptor. Science 1988;241:825-828

16. Pittenger MF, Mackay AM, Beck SC, Jaiswal RK, Douglas R, Mosca JD, Moorman MA, Simonetti DW, Craig S, Marshak DR. Multilineage potential of adult human mesenchymal stem cells. Science 1999;284:143-147

17. Barry FP, Boynton RE, Haynesworth S, Murphy JM, Zaia J. The monoclonal antibody $\mathrm{SH}-2$, raised against human mesenchymal stem cells, recognizes an epitope on endoglin (CD105). Biochem Biophys Res Commun 1999;265:134-139

18. Kyriakis JM, Avruch J. Mammalian MAPK signal transduction pathways activated by stress and inflammation: a 10-year update. Physiol Rev 2012;92:689-737

19. Legendre F, Bogdanowicz P, Boumediene K, Pujol JP. Role of interleukin 6 (IL-6)/IL-6R-induced signal tranducers and activators of transcription and mitogen-activated protein kinase/extracellular. J Rheumatol 2005;32:1307-1316

20. Chen JD, Xu FF, Zhu H, Li XM, Tang B, Liu YL, Zhang Y. [ICAM-1 regulates differentiation of MSC to adipocytes via activating MAPK pathway]. Zhongguo Shi Yan Xue Ye Xue Za Zhi 2014;22:160-165. Chinese

21. Pricola KL, Kuhn NZ, Haleem-Smith H, Song Y, Tuan RS. Interleukin-6 maintains bone marrow-derived mesenchymal stem cell stemness by an ERK1/2-dependent mechanism. J Cell Biochem 2009;108:577-588

22. Aouadi M, Jager J, Laurent K, Gonzalez T, Cormont M, Binétruy B, Le Marchand-Brustel Y, Tanti JF, Bost F. p38MAP Kinase activity is required for human primary adipocyte differentiation. FEBS Lett 2007;581:5591-5596

23. Aouadi M, Laurent K, Prot M, Le Marchand-Brustel Y, Binétruy B, Bost F. Inhibition of p38MAPK increases adipogenesis from embryonic to adult stages. Diabetes 2006; 55:281-289

24. Batchvarova N, Wang XZ, Ron D. Inhibition of adipogenesis by the stress-induced protein CHOP (Gadd153). EMBO J 1995;14:4654-4661

25. Sonowal H, Kumar A, Bhattacharyya J, Gogoi PK, Jaganathan BG. Inhibition of actin polymerization decreases osteogeneic differentiation of mesenchymal stem cells through p38 MAPK pathway. J Biomed Sci 2013;20:71

26. Martin $\mathrm{AD}$, Daniel MZ, Drinkwater DT, Clarys JP. Adipose tissue density, estimated adipose lipid fraction and whole body adiposity in male cadavers. Int J Obes Relat Metab Disord 1994;18:79-83

27. Prior BM, Cureton KJ, Modlesky CM, Evans EM, Sloniger MA, Saunders M, Lewis RD. In vivo validation of whole body composition estimates from dual-energy X-ray absorptiometry. J Appl Physiol (1985) 1997;83:623-630

28. Matsushita K. Mesenchymal stem cells and metabolic syndrome: current understanding and potential clinical implications. Stem Cells Int 2016;2016:2892840 